\title{
Abstract intersection theory and operators in Hilbert space
}

\author{
Grzegorz BAnAszaK and YoICHi Uetake
}

\begin{abstract}
For an operator in Hilbert space of a certain class, we introduce axioms of an abstract intersection theory, which we prove to be equivalent to the Riemann hypothesis regarding the spectrum of that operator. In particular, if the nontrivial zeros of the Riemann zeta-function arise from an operator of this class, the original Riemann hypothesis is equivalent to the existence of an abstract intersection theory.
\end{abstract}

\section{Introduction}

Let $A$ be a linear operator acting on a Hilbert space $H$ such that its spectrum $\sigma(A)$ consists only of the point spectrum $\sigma_{p}(A)$ (i.e., eigenvalues). We say that the operator $A$ satisfies the Riemann hypothesis (RH, shortly) if $\operatorname{Re}(s)=\frac{1}{2}$ for all $s \in \sigma(A)=\sigma_{p}(A)$.

We introduce a set of axioms ((INT1) to (INT3) in Section 3.1), which we show to be equivalent to the $\mathrm{RH}$ for the operator $A$. The axioms constitute a theory that is analogous to Weil's classical intersection theory on a surface over a finite field $\mathbb{F}_{q}$ and to his explicit formulae [12] (see also $[4,6,10]$ ). Recall that Weil developed his intersection theory to prove the $\mathrm{RH}$ for curves over $\mathbb{F}_{q}$. In this regard, we call our axioms an abstract intersection theory.

The paper is organized as follows. In Section 2, we impose some reasonable conditions (OP1) to (OP5) on operators in Hilbert space to be considered. Then we introduce a functional calculus for them, which has a role of cutting off their spectra. Our abstract intersection theory consists of conditions (INT1) to (INT2) on some specific vectors including what we call a Hodge vector, and the Lefschetz type formula (INT3). We describe this in Section 3.1. In Section 3.2, we give a model of the abstract intersection theory, using a construction similar to the GNS (Gelfand-Naimark-Segal) representation (e.g., [9]). Interestingly, Weil himself reviewed Segal's work [9] in the Mathematical Reviews. Using this model, we show in Theorem 3.1 (main theorem) that the $\mathrm{RH}$ for the operator $A$ is equivalent to the existence of an intersection theory in our sense. 
One of the key ideas of our paper is that an $\mathbb{R}$-linear structure is introduced in the abstract intersection theory while an operator regarding $\mathrm{RH}$ acts on a $\mathbb{C}$-Hilbert space. We also introduce $\mathbb{R}$-valued functions $q=q(Y)$ and $g=g(Y)$ of the $Y$-coordinate of the critical strip. These can be seen as analogs of $q=\sharp \mathbb{F}_{q}$ and the genus number $g$ of a curve $C$, respectively, in the classical intersection theory on a surface $C \times C$, which was used to prove the $\mathrm{RH}$ for $C$ over $\mathbb{F}_{q}$. Moreover, we introduce an operator valued function $\Phi=\Phi(Y)$, which is an analog of the operator acting on the Picard group of $C \times C$ induced by the classical Frobenius morphism. For further comparison of our constructions with the classical theory, see Section 4.

We note that there is a program by Connes and Marcolli [1] to adapt Weil's proof to the case of number fields. There is also a conjectural cohomology theory by Deninger [2] for the interpretation of $L$-functions analogous to the etale cohomology theory of varieties over finite fields.

\section{Functional calculus for closed operators}

Let $A: H \supset \operatorname{dom}(A) \rightarrow H$ be a possibly unbounded operator on a separable $\mathbb{C}$-Hilbert space $H$. We assume the following properties of $A$.

(OP1) $A$ is closed.

(OP2) The spectrum $\sigma(A)$ consists only of the point spectrum (i.e., eigenvalues) $\sigma_{p}(A)$ (i.e., $\sigma(A)=\sigma_{p}(A)$ ), which accumulates at most at infinity.

(OP3) (a) Image $\left(P_{\left\{s_{\alpha}\right\}}\right)$ (see Lemma 2.1 below for definition) is finite dimensional for any $s_{\alpha} \in \sigma_{p}(A)$.

(b) The Riesz index $\nu\left(s_{\alpha}\right)=1$ for any $s_{\alpha} \in \sigma_{p}(A)$. (See the paragraph following Lemma 2.1 for definition.)

(OP4) $\sigma(A) \subset \Omega_{\infty}$, where $\Omega_{\infty}:=\{s \in \mathbb{C} ; 0<\operatorname{Re}(s)<1\}$.

(OP5) (a) $\operatorname{Re}\left(s_{\alpha}\right)<\frac{1}{2}$ for some $s_{\alpha} \in \sigma(A)$ if and only if there is $s_{\beta} \in \sigma(A)$ such that $\operatorname{Re}\left(s_{\beta}\right)>\frac{1}{2}$.

(b) If $s_{\alpha} \in \sigma(A)$ then $\overline{s_{\alpha}} \in \sigma(A)$ with the same multiplicity $\operatorname{mult}\left(\overline{s_{\alpha}}\right)=\operatorname{mult}\left(s_{\alpha}\right)$. (See the paragraph following Lemma 2.1 for definition.)

Automorphic scattering theory can provide a closed operator that satisfies the above conditions except for (OP3-b). The operator of this theory gives a spectral interpretation of the nontrivial zeros of the Dirichlet 
$L$-function. Hinted by Gelfand, the theory was initiated by Pavlov and Faddeev [7] using the Lax-Phillips scattering theory, and refined by Lax and Phillips themselves [5]. Recently, in [11, Theorem 4.1], directly based on the properties of Eienstein series over a modular curve, an algebraic method of construction of a Dirac-type operator $A_{0}$ for automorphic scattering that satisfies (OP1) to (OP5) except for (OP3-b) has been developed for Dirichlet $L$-functions including the Riemann zeta-function. The eigenvalues of the operator $A_{0}$ in [11] are shown to correspond, counted with multplicity, to the nontrivial zeros of the associated Dirichlet $L$-function. The construction in [11] does not appeal to either RH or GRH. The operator $A_{0}$ satisfies also (OP3-b) if and only if the multiplicity of nontrivial zeros of the $L$-function corresponding to $A_{0}$ constructed there is one (Theorem 4.1 (iii), (iv) of [11]). See Corollary 3.1 in Section 3 for our result concerning the operator $A_{0}$.

By (OP1) we can use the following lemma from [3, XV.2, Theorem 2.1, p. 326].

Lemma 2.1. Suppose that $A: H \supset \operatorname{dom}(A) \rightarrow H$ is a closed operator. For a bounded subset $W$ of $\sigma(A)$, let $P_{W}: H \rightarrow H$ be the Riesz projection

$$
P_{W}=\frac{1}{2 \pi i} \oint_{\partial \Delta}(s-A)^{-1} d s,
$$

where $\Delta$ is a bounded domain of $\mathbb{C}$ such that $W \Subset \Delta$ (i.e., $\bar{W} \subset \Delta^{\circ}$ ) and $\bar{\Delta} \cap \tau=\emptyset$ for $\tau=\sigma(A) \backslash W$. Then

(i) $M=\operatorname{Image}\left(P_{W}\right)$ and $N=\operatorname{Ker}\left(P_{W}\right)$ are $A$-invariant (i.e. $A(N \cap \operatorname{dom}(A)) \subset N$ etc. $)$.

(ii) $M \subset \operatorname{dom}(A)$ and $\left.A\right|_{M}$ (the restriction of $A$ to $M$ ) is bounded.

(iii) $\sigma\left(\left.A\right|_{M}\right)=W$ and $\sigma\left(\left.A\right|_{N}\right)=\tau$.

By (OP3) and Lemma 2.1 (i) and (iii), the resolvent $(s-A)^{-1}$ has a pole of order $\nu\left(s_{\alpha}\right)=1$ at $s=s_{\alpha} \in \sigma_{p}(A)$. Here $\nu\left(s_{\alpha}\right)$ denotes the Riesz index of $s_{\alpha}$ defined as the smallest positive integer such that $\operatorname{Ker}\left(\left(s_{\alpha}-A\right)^{\nu\left(s_{\alpha}\right)}\right)=$ $\operatorname{Image}\left(P_{\left\{s_{\alpha}\right\}}\right)$. We call mult $\left(s_{\alpha}\right):=\operatorname{dim} \operatorname{Image}\left(P_{\left\{s_{\alpha}\right\}}\right)$ the (algebraic) multiplicity of $s_{\alpha}$. By (OP2), $(s-A)^{-1}$ is meromorphic in $\mathbb{C}$.

For $Y>0$ let $\sigma_{Y}(A):=\{s \in \sigma(A) ;|\operatorname{Im}(s)| \leq Y\}$. By (OP2) one can take some $\epsilon_{Y}>0$ and $\Omega_{Y}=\left\{s \in \mathbb{C} ; 0<\operatorname{Re}(s)<1,|\operatorname{Im}(s)|<Y+\epsilon_{Y}\right\}$ so that $\overline{\Omega_{Y}} \cap\left(\sigma(A) \backslash \sigma_{Y}(A)\right)=\emptyset$. Note that $\Omega_{Y} \cap \sigma(A)=\sigma_{Y}(A)$ by (OP4).

Let $\mathcal{F}$ be an $\mathbb{R}$-algebra defined by $\mathcal{F}:=\left\{\phi ; \phi(s)\right.$ is analytic in an open set $\ni \Omega_{\infty}$ and $\left.\overline{\phi(\bar{s})}=\phi(s)\right\}$. 
Note that no topological structure is imposed on $\mathcal{F}$.

Given $\phi \in \mathcal{F}$, let $\phi(A): H \supset \operatorname{dom}(\phi(A)) \rightarrow H$ be defined by

$$
\phi(A) x=\lim _{Y \rightarrow \infty} \frac{1}{2 \pi i}\left(\oint_{\partial \Omega_{Y}} \phi(s)(s-A)^{-1} d s\right) x
$$

for $x \in \operatorname{dom}(\phi(A)):=\{x \in H$; the limit $\phi(A) x$ exists in $H\}$. By (OP3-b), Lemma 2.1 and the functional calculus for bounded operators, we have $\phi(A)=\sum_{s_{\alpha} \in \sigma(A)} \phi\left(s_{\alpha}\right) P_{\left\{s_{\alpha}\right\}}$.

We define $\operatorname{tr}(\phi(A))$ as a functional on $\mathcal{F}$ as follows: define

$$
\operatorname{tr}(\cdot(A)): \mathcal{F} \supset \operatorname{dom}(\operatorname{tr}(\cdot(A))) \rightarrow \mathbb{C}
$$

by

$$
\operatorname{tr}(\phi(A))=\sum_{s_{\alpha} \in \sigma(A)} \operatorname{mult}\left(s_{\alpha}\right) \phi\left(s_{\alpha}\right)
$$

where

$$
\operatorname{dom}(\operatorname{tr}(\cdot(A))):=\left\{\phi \in \mathcal{F} ; \sum_{s_{\alpha} \in \sigma(A)} \operatorname{mult}\left(s_{\alpha}\right) \phi\left(s_{\alpha}\right)<\infty\right\}
$$

Let $q: \mathcal{F} \rightarrow \mathbb{R}$ be defined by $q(\phi)=\phi(1)$.

Lemma 2.2. Suppose that $q_{Y} \in(0,1) \cup(1, \infty)$ is given for each $Y>0$. Then for each $Y>0$ there is a $\phi_{Y} \in \mathcal{F}$ such that

(i) $\phi_{Y}(0)=1$,

(ii) $q\left(\phi_{Y}\right)=q_{Y}$,

(iii) $\phi_{Y}(s)=q_{Y}^{s}$, if $s \in \sigma_{Y}(A)$,

(iv) $\phi_{Y}(s)=0$, if $s \in \sigma(A) \backslash \sigma_{Y}(A)$.

Proof. Let

$$
\left\{s_{n}\right\}_{n=1}^{N}:=\left[\sigma(A) \backslash \sigma_{Y}(A)\right] \cap\{s \in \mathbb{C} ; \operatorname{Im}(s)>0\}(N \leq \infty) .
$$

Note that we have $N=\infty$ if the operator $A$ has an infinite spectrum. Let $h_{Y}(s)$ be an entire function which has zeros of order one only at $s_{n}$ and $\overline{s_{n}}$ 
$(1 \leq n \leq N)$. For example, one can set

$$
h_{Y}(s):=\prod_{n=1}^{N} E_{n-1}\left(\frac{s}{s_{n}}\right) E_{n-1}\left(\frac{s}{\overline{s_{n}}}\right)
$$

where $E_{0}(s)=1-s$ and $E_{p}(s)=(1-s) \exp \left(\sum_{k=1}^{p} \frac{s^{k}}{k}\right)$ for $p \geq 1$ (see e.g. [8, Theorem 15.9, p. 324]). Then by (OP5-b) $h_{Y}\left(s_{\alpha}\right)=0$ if and only if $s_{\alpha} \in$ $\sigma(A) \backslash \sigma_{Y}(A)$. Note that $\overline{h_{Y}(\bar{s})}=h_{Y}(s)$. Observe that $\sigma_{Y}(A)$ is a finite set by (OP2) and (OP4). Thus one can take a Lagrange polynomial $\ell_{Y}(s)$ so that $\ell_{Y}(0)=\frac{1}{h_{Y}(0)}, \ell_{Y}(1)=\frac{q_{Y}}{h_{Y}(1)}$, and $\ell_{Y}\left(s_{\alpha}\right)=\frac{q_{Y}^{s_{\alpha}}}{h_{Y}\left(s_{\alpha}\right)}$ if $s_{\alpha} \in \sigma_{Y}(A)$. Note that by (OP5-b) we have $\overline{\ell_{Y}(\bar{s})}=\ell_{Y}(s)$. Then $\phi_{Y}(s):=\ell_{Y}(s) h_{Y}(s)$ is a desired function.

Although it seems that the information of the spectrum of $A$ is needed to calculate $\phi_{Y}(A), \phi_{Y}(A) x$ is actually obtained from (OP4) as

$$
\phi_{Y}(A) x=\frac{1}{2 \pi i}\left(\oint_{\partial \Omega_{Y}} q_{Y}^{s}(s-A)^{-1} d s\right) x \text { for all } x \in H
$$

provided that one can take $\epsilon_{Y}=0$ in the definition of $\Omega_{Y}$ (This is generically possible). From this expression we see that $\phi_{Y}(A)$ is bounded.

We define $g: \mathcal{F} \rightarrow[0, \infty]$ by $g(\phi)=\frac{1}{2} \operatorname{dim} \operatorname{Image}(\phi(A))$. Note that $g\left(\phi_{Y}\right)<\infty$ for each $Y>0$. Let us write

$$
q=q(Y):=q\left(\phi_{Y}\right)\left(=q_{Y}\right) \text { and } g=g(Y):=g\left(\phi_{Y}\right)
$$

\section{Abstract intersection theory}

\subsection{Axioms of abstract intersection theory}

Let $V$ be an $\mathbb{R}$-linear space, endowed with a symmetric bilinear form $\beta: V \times$ $V \rightarrow \mathbb{R}$. Let $\operatorname{End}_{\mathbb{R}}(V)$ denote the $\mathbb{R}$-algebra of $\mathbb{R}$-linear operators on $V$. Suppose that there are nonzero vectors $v_{01}, v_{10}$ and $h_{a}$ in $V$, a mapping $v_{\delta}: \mathcal{F} \supset \operatorname{dom}\left(v_{\delta}\right) \rightarrow V$, and an $\mathbb{R}$-algebra homomorphism $\Phi: \mathcal{F} \supset \operatorname{dom}(\Phi) \rightarrow$ $\operatorname{End}_{\mathbb{R}}(V)$ that satisfy the conditions listed below, which we call an abstract intersection theory. $v_{\delta}$ and $\Phi$ are associated with the operator $A$ in Section 2. We assume that $\operatorname{dom}\left(v_{\delta}\right)=\operatorname{dom}(\Phi)=\operatorname{dom}(\operatorname{tr}(\cdot(A)))$ and that it is an $\mathbb{R}$-subalgebra of $\mathcal{F}$. We also assume that $\phi_{Y} \in \mathcal{F}$ introduced in Lemma 2.2 belongs to $\operatorname{dom}\left(v_{\delta}\right)$. Let us write

$$
v_{\delta}=v_{\delta}(Y):=v_{\delta}\left(\phi_{Y}\right) \text { and } \Phi=\Phi(Y):=\Phi\left(\phi_{Y}\right)
$$


(INT1) (a) $\beta(y, x)=\beta(x, y) \in \mathbb{R}$ for $x, y \in V$,

(b) $\beta\left(v_{01}, v_{01}\right)=0, \quad$ (c) $\beta\left(v_{10}, v_{10}\right)=0, \quad$ (d) $\beta\left(v_{01}, v_{10}\right)=1$,

(e) $\beta\left(\Phi^{n} v_{\delta}, v_{01}\right)=1$, (f) $\beta\left(\Phi^{n} v_{\delta}, v_{10}\right)=O\left(q^{n}\right)$,

(g) $\beta\left(\Phi^{n} v_{\delta}, \Phi^{n} v_{\delta}\right)=O\left(q^{n}\right)$.

(INT2) For $x \in V$, if $\beta\left(x, h_{a}\right)=0$ then $\beta(x, x) \leq 0$.

Note that (INT1) is assumed to hold for each $Y>0$. The Bachmann-Landau notation $O\left(q^{n}\right)$ in (INT1) is with respect to $n \gg 0$ for $q=q(Y)$ fixed. We call (INT2) the Hodge property, and $h_{a}$ a Hodge vector.

Lemma 3.1. Under the assumptions (INT1-a) to (INT1-d) and (INT2), we have

$$
\beta(x, x) \leq 2 \beta\left(x, v_{01}\right) \beta\left(x, v_{10}\right) \quad(x \in V) .
$$

Proof. Given any $x \in V$, define ${ }^{\wedge}: \mathbb{R}^{3} \rightarrow V$ by $\hat{r}=r_{1} v_{01}+r_{2} v_{10}+r_{3} x$ for $r=\sum_{j=1}^{3} r_{j} e_{j}$. Here $e_{1}=(1,0,0)^{t}, e_{2}=(0,1,0)^{t}$ and $e_{3}=(0,0,1)^{t}$. Let $E_{1}=e_{1}+e_{2}$ and $E_{2}=e_{1}-e_{2}$. Then by (INT1-b) to (INT1-d), $\beta\left(\hat{E}_{1}, \hat{E}_{1}\right)=$ $2, \beta\left(\hat{E}_{2}, \hat{E}_{2}\right)=-2$ and $\beta\left(\hat{E}_{1}, \hat{E}_{2}\right)=0$. Let $E_{3}=e_{3}+k_{1} E_{1}+k_{2} E_{2}$. Then

$$
\begin{aligned}
& \beta\left(\hat{E}_{3}, \hat{E}_{1}\right)=\beta\left(x, v_{01}\right)+\beta\left(x, v_{10}\right)+2 k_{1} \text { and } \\
& \beta\left(\hat{E}_{3}, \hat{E}_{2}\right)=\beta\left(x, v_{01}\right)-\beta\left(x, v_{10}\right)-2 k_{2} .
\end{aligned}
$$

Hence one can set

$$
k_{1}=-\frac{1}{2}\left\{\beta\left(x, v_{01}\right)+\beta\left(x, v_{10}\right)\right\} \quad \text { and } \quad k_{2}=\frac{1}{2}\left\{\beta\left(x, v_{01}\right)-\beta\left(x, v_{10}\right)\right\}
$$

so that $\beta\left(\hat{E}_{3}, \hat{E}_{1}\right)=\beta\left(\hat{E}_{3}, \hat{E}_{2}\right)=0$. Then one can check that

$$
\beta\left(\hat{E}_{3}, \hat{E}_{3}\right)=\beta(x, x)-2 \beta\left(x, v_{01}\right) \beta\left(x, v_{10}\right) .
$$

Now suppose $\beta\left(\hat{E}_{3}, \hat{E}_{3}\right)>0$. Then $m:=\beta\left(\hat{E}_{3}, h_{a}\right) \neq 0$ by the Hodge property in (INT2) and for $n:=-\beta\left(\hat{E}_{1}, h_{a}\right)$,

$$
\begin{aligned}
\beta\left(m \hat{E}_{1}+n \hat{E}_{3}, m \hat{E}_{1}+n \hat{E}_{3}\right) & =m^{2} \beta\left(\hat{E}_{1}, \hat{E}_{1}\right)+n^{2} \beta\left(\hat{E}_{3}, \hat{E}_{3}\right) \geq m^{2} \beta\left(\hat{E}_{1}, \hat{E}_{1}\right) \\
& =2 m^{2}>0 .
\end{aligned}
$$

But we have $\beta\left(m \hat{E}_{1}+n \hat{E}_{3}, h_{a}\right)=0$, which contradicts the Hodge property. Hence we get the claim. 
For $x, y \in V$ let

$$
\langle x, y\rangle_{V}=\beta\left(x, v_{01}\right) \beta\left(y, v_{10}\right)+\beta\left(x, v_{10}\right) \beta\left(y, v_{01}\right)-\beta(x, y)
$$

By Lemma 3.1, $\langle\cdot, \cdot\rangle_{V}$ is positive semidefinite, i.e., $\langle x, x\rangle_{V} \geq 0$ for $x \in V$. Indeed, as we will see soon below, this bilinear form must be positive semidefinite, not positive definite.

It is easy to see that from (INT1) and (*) the following conditions follow.

(IP) (a) $\langle y, x\rangle_{V}=\langle x, y\rangle_{V} \in \mathbb{R}$ for $x, y \in V$.

(b) $\left\langle v_{01}, v_{01}\right\rangle_{V}=0 . \quad$ (c) $\left\langle v_{10}, v_{10}\right\rangle_{V}=0 . \quad$ (d) $\left\langle v_{01}, v_{10}\right\rangle_{V}=0$.

(e) $\left\langle\Phi^{n} v_{\delta}, v_{01}\right\rangle_{V}=0$. (f) $\left\langle\Phi^{n} v_{\delta}, v_{10}\right\rangle_{V}=0$.

(g) $\left\langle\Phi^{n} v_{\delta}, \Phi^{n} v_{\delta}\right\rangle_{V}=O\left(q^{n}\right)$.

Here $v_{\delta}, \Phi$ and $q$ are parameterized by $Y$ as in (INT1).

From the positive semidefinite property, we obtain the Cauchy-Schwarz inequality:

$$
\left|\langle x, y\rangle_{V}\right| \leq \sqrt{\langle x, x\rangle_{V}\langle y, y\rangle_{V}}, \quad(x, y \in V)
$$

Note that by this inequality, $\left\langle x, y_{0}\right\rangle_{V}=0$ for all $x \in V$ if $\left\langle y_{0}, y_{0}\right\rangle_{V}=0$. Accordingly, $\left\langle x, v_{01}\right\rangle_{V}=\left\langle x, v_{10}\right\rangle_{V}=0$ for $x \in V$.

Now we introduce axiom (INT3), which we call the Lefschetz type formula.

(INT3) For any $\phi \in \operatorname{dom}\left(v_{\delta}\right)$ and any $n \geq 0$,

$$
\operatorname{tr}\left(\phi(A)^{n}\right)=\left\langle\Phi(\phi)^{n} v_{\delta}(\phi), v_{\delta}(\phi)\right\rangle_{V}
$$

\subsection{A model of abstract intersection theory and main theorem}

The following construction, which we call a model of abstract intersection theory, is hinted by the GNS (Gelfand-Naimark-Segal) construction [9].

Let $\left\{e_{j}\right\}_{j=1}^{\operatorname{dim} H} \quad(1 \leq \operatorname{dim} H \leq \infty)$ be a complete orthonormal basis of $H$. Embed $H$ into a bigger Hilbert space $K$, so that $K \ominus H=\mathbb{C}^{2}$. Here $K \ominus H$ is the orthogonal complement of $H$ in $K$. In our convention the inner product on $X=H$ or $\mathbb{C}^{2}$ satisfies the equality $\left\langle\lambda h_{1}, h_{2}\right\rangle_{X}=\lambda\left\langle h_{1}, h_{2}\right\rangle_{X}$. Hence we have $\left\langle\lambda k_{1}, k_{2}\right\rangle_{K}=\lambda\left\langle k_{1}, k_{2}\right\rangle_{K}$. We understand that $e_{j}$ is embedded in $K$ as 
$e_{j} \mapsto e_{j}^{\prime}=\left(\begin{array}{c}e_{j} \\ 0 \\ 0\end{array}\right)$. Let $B(K)$ denote a set of bounded operators on $K$. Put

$$
V_{1}=\left\{x \in B(K) ;\|x\|_{V_{1}}^{2}:=\sum_{j=1}^{\operatorname{dim} H}\left\langle x^{*} x e_{j}^{\prime}, e_{j}^{\prime}\right\rangle_{K}<\infty\right\}
$$

as an $\mathbb{R}$-linear space of Hilbert-Schmidt type class with a semidefinite inner product $\langle x, y\rangle_{V_{1}}=\frac{1}{2} \sum_{j=1}^{\operatorname{dim} H}\left\langle\left(y^{*} x+x^{*} y\right) e_{j}^{\prime}, e_{j}^{\prime}\right\rangle_{K}$ for $x, y \in V_{1}$.

Define some elements of $V_{1}$ in block diagonal form (acting on $K=$ $\underset{K}{\stackrel{H}{\ominus} \underset{\ominus}{\ominus}}$ ) as follows (blank $=0)$ :

$$
\begin{aligned}
& v_{01}:=\left(\begin{array}{c|cc}
0 & & \\
\hline & 0 & 1 \\
& 0 & 0
\end{array}\right), \quad v_{10}:=\left(\begin{array}{c|cc}
0 & & \\
\hline & 0 & 0 \\
& 1 & 0
\end{array}\right), \\
& v_{\delta 1}(\phi):=\left(\begin{array}{c|cc}
P_{H \phi}^{*} P_{H^{\phi}} & & \\
\hline & 0 & 0 \\
& 0 & 0
\end{array}\right), \\
& v_{\delta}(\phi):=v_{\delta 1}(\phi)+v_{01}+v_{10}\left(\phi \in \operatorname{dom}\left(v_{\delta}\right)\right) \text {. }
\end{aligned}
$$

Here $H^{\phi}:=\operatorname{Image}(\phi(A))$ and $P_{H^{\phi}}: H \rightarrow H^{\phi}$ denotes the orthogonal projection of $H$ onto $H^{\phi}$ (not a Riesz projection in Lemma 2.1). In this model of abstract intersection theory we let

$$
\begin{aligned}
\operatorname{dom}\left(v_{\delta}\right) & =\operatorname{dom}(\Phi)=\operatorname{dom}(\operatorname{tr}(\cdot(A))) \\
& :=\left\{\phi \in \mathcal{F} ; \phi \text { has zeros at all but a finite number of } s_{\alpha} \in \sigma(A)\right\}
\end{aligned}
$$

If $\phi \in \operatorname{dom}\left(v_{\delta}\right)$ then $g(\phi)<\infty$. Note that $\phi_{Y} \in \operatorname{dom}\left(v_{\delta}\right)$. It is easy to see that $v_{01}, v_{10}$ belong to $V_{1}$, and that $v_{\delta}(\phi) \in V_{1}$ for $\phi \in \operatorname{dom}\left(v_{\delta}\right)$.

Lemma 3.2. Suppose that an operator $A: H \supset \operatorname{dom}(A) \rightarrow H$ that satisfies (OP1) to (OP4) and (OP-5b) is given. Let $\phi_{Y}(Y>0)$ be as defined in Section 2. Then for the above $\mathbb{R}$-linear space $V_{1}$ there exists an $\mathbb{R}$-algebra homomorphism $\Phi: \mathcal{F} \supset \operatorname{dom}\left(v_{\delta}\right) \rightarrow \operatorname{End}_{\mathbb{R}}\left(V_{1}\right)$, so that

(i) The conditions (IP-a) to (IP-f) with $V$ replaced by $V_{1}$ hold.

(ii) The Lefschetz type formula (INT3) with $V$ replaced by $V_{1}$ holds.

Proof. (i) (IP-a) is obvious from definition. Define $\Phi: \mathcal{F} \supset \operatorname{dom}\left(v_{\delta}\right) \rightarrow$ $\operatorname{End}_{\mathbb{R}}\left(V_{1}\right)$ by

$$
\Phi(\phi) x=\left(\begin{array}{c|cc}
\phi(A) & & \\
\hline & \phi(1) & 0 \\
& 0 & \phi(0)
\end{array}\right) x
$$


for $\phi \in \operatorname{dom}\left(v_{\delta}\right)$ and $x \in V_{1}$. It is easy to check that $\Phi(\phi) x \in V_{1}$ if $x \in V_{1}$. Note that $\operatorname{dom}\left(v_{\delta}\right)$ is an $\mathbb{R}$-subalgebra of $\mathcal{F}$. Observe that if $\phi_{1}, \phi_{2} \in \operatorname{dom}\left(v_{\delta}\right)$ then $\phi_{1}(A)$ and $\phi_{2}(A)$ are bounded, and that $\left(\phi_{1} \phi_{2}\right)(A)=\phi_{1}(A) \phi_{2}(A)$. Hence one can easily see that $\Phi$ is a well-defined $\mathbb{R}$-algebra homomorphism. It is easy to check that $\Phi(\phi)^{n} v_{\delta}(\phi) \in V_{1}(n \geq 0)$ provided that $\phi \in \operatorname{dom}\left(v_{\delta}\right)$. We recall that $\phi_{Y} \in \operatorname{dom}\left(v_{\delta}\right)$. It is also easy to see that the vectors $v_{01}, v_{10}$ and $v_{\delta}$ satisfy the conditions (IP-b) to (IP-f). For example, $\left\langle v_{01}, v_{01}\right\rangle_{V_{1}}=$ $\sum_{j=1}^{\operatorname{dim} H}\left\langle v_{01}^{*} v_{01} e_{j}^{\prime}, e_{j}^{\prime}\right\rangle_{K}$. However, since $v_{01} e_{j}=0$ one gets (IP-b). To check (IP-e) and (IP-f) note that

$$
\Phi(\phi)^{n} v_{\delta}(\phi)=\Phi(\phi)^{n} v_{\delta 1}(\phi)+\phi(1)^{n} v_{01}+\phi(0)^{n} v_{10}
$$

(ii) For $\phi \in \operatorname{dom}\left(v_{\delta}\right)$ let $\left\{e_{j}\right\}_{j=1}^{2 g(\phi)}$ be an orthonormal basis of $H^{\phi}$. Then, since $\phi(A) H^{\phi} \subset H^{\phi}$, we have

$$
\begin{aligned}
\left\langle\Phi(\phi)^{n} v_{\delta}(\phi), v_{\delta}(\phi)\right\rangle_{V_{1}} & =\frac{1}{2} \sum_{j=1}^{2 g(\phi)}\left\{\left\langle\phi(A)^{n} e_{j}, e_{j}\right\rangle_{H^{\phi}}+\left\langle\phi(A)^{* n} e_{j}, e_{j}\right\rangle_{H^{\phi}}\right\} \\
& =\frac{1}{2} \sum_{j=1}^{2 g(\phi)}\left\{\left\langle\phi(A)^{n} e_{j}, e_{j}\right\rangle_{H^{\phi}}+\overline{\left\langle\phi(A)^{n} e_{j}, e_{j}\right\rangle_{H^{\phi}}}\right\} \\
& =\frac{1}{2}\left(\operatorname{tr}\left(\phi(A)^{n}\right)+\overline{\operatorname{tr}\left(\phi(A)^{n}\right)}\right),
\end{aligned}
$$

which is $\operatorname{tr}\left(\phi(A)^{n}\right)$ provided that $\operatorname{tr}\left(\phi(A)^{n}\right) \in \mathbb{R}$. This condition is satisfied by $(\mathrm{OP} 5-\mathrm{b})$ and the definition of $\mathcal{F}$.

The following lemma says that given $V_{1}$ as above, one can find many $V$ 's and $\beta$ 's satisfying (INT1) and (INT2).

Lemma 3.3. In the same situation as in Lemma 3.2 and its proof, suppose that $\Phi=\Phi(Y)\left(=\Phi\left(\phi_{Y}\right)\right)$ further satisfies (IP-g). Let $V$ be an $\mathbb{R}$-linear subspace of $V_{1}$ such that $v_{01}, v_{10}$ and $\Phi^{n} v_{\delta}=\Phi(Y)^{n} v_{\delta}(Y)$ all belong to $V$ for any $Y>0$.

Then there is a bilinear form $\beta: V \times V \rightarrow \mathbb{R}$ and a Hodge vector $h_{a} \in V$ which satisfy (INT1) and (INT2) and (*).

Proof. (INT1) and $(*)$ : In $(*)$ let $\langle\cdot, \cdot\rangle_{V}$ be the inner product on $V$ inherited from $\langle\cdot, \cdot\rangle_{V_{1}}$. Given $\langle\cdot, \cdot\rangle_{V}$, one can determine $\beta(x, y)$ from $\beta\left(x, v_{01}\right)$, $\beta\left(x, v_{10}\right), \beta\left(y, v_{01}\right)$ and $\beta\left(y, v_{10}\right)$ via $(*)$. Decompose $V$ into a (not necessarily orthogonal) direct sum of $W_{1}$ and $W_{2}$, where $W_{1}$ is the $\mathbb{R}$-linear span of $\left\{v_{01}, v_{10}, \Phi(Y)^{n} v_{\delta}(Y) ; Y>0, n \geq 0\right\}$. 
Let us write $v_{\delta 1}=v_{\delta 1}(Y):=v_{\delta 1}\left(\phi_{Y}\right)$. Note that

$$
\operatorname{span}_{\mathbb{R}}\left\{\Phi(Y)^{n} v_{\delta 1}(Y): Y>0, n \geq 0\right\} \cap \operatorname{span}_{\mathbb{R}}\left\{v_{01}, v_{10}\right\}=\{0\}
$$

Hence one can define $\beta\left(\cdot, v_{01}\right), \beta\left(\cdot, v_{10}\right), \beta\left(v_{01}, \cdot\right)$ and $\beta\left(v_{10}, \cdot\right)$ on $W_{1}$ so as to satisfy (INT1-a) to (INT1-d) and

$$
\beta\left(\Phi^{n} v_{\delta 1}, v_{01}\right)=0(n \geq 0), \quad \beta\left(\Phi^{n} v_{\delta 1}, v_{10}\right)=0(n \geq 0) .
$$

Then, since $\Phi^{n} v_{\delta}=\Phi^{n} v_{\delta 1}+q^{n} v_{01}+v_{10}$ for all $n \geq 0$, we see that $\beta$ satisfies (INT1-e) and (INT1-f). (INT1-g) also follows from (IP-g), (INT1-e) and (INT1-f) via $(*)$.

Assign arbitrary $\mathbb{R}$-linear mappings $\beta\left(\cdot, v_{01}\right)$ and $\beta\left(\cdot, v_{10}\right)$ of $W_{2}$ to $\mathbb{R}$. Then one can determine $\beta$ on $V \times V$ via $(*)$. One can check (INT1-a) since (IP-a) holds in $(*)$.

(INT2): Let $h_{a}=v_{01}+v_{10}$. If $\beta\left(x, h_{a}\right)=0$, then $\beta\left(x, v_{10}\right)=-\beta\left(x, v_{01}\right)$. Thus $\beta(x, x)=2 \beta\left(x, v_{01}\right) \beta\left(x, v_{10}\right)-\langle x, x\rangle_{V}=-2 \beta\left(x, v_{01}\right)^{2}-\langle x, x\rangle_{V} \leq 0$. Therefore $h_{a}$ is a Hodge vector.

We use the following lemma (e.g. [6, Lemma 2.2, p.20]) in the proof of Theorem 3.1 below.

Lemma 3.4. Let $\lambda_{j}(1 \leq j \leq 2 g)$ be complex numbers. Then there exist infinitely many integers $n \geq 1$ such that $\left|\lambda_{1}\right|^{n} \leq\left|\sum_{j=1}^{2 g} \lambda_{j}^{n}\right|$.

Theorem 3.1. Let $A: H \supset \operatorname{dom}(A) \rightarrow H$ be an operator satisfying (OP1) to (OP5). The following conditions are equivalent.

(i) The Riemann hypothesis holds for $A$.

(ii) There exist an $\mathbb{R}$-linear space $V$, a symmetric bilinear $\mathbb{R}$-valued form $\beta$ on $V$, a mapping $v_{\delta}$ of $\operatorname{dom}\left(v_{\delta}\right) \subset \mathcal{F}$ into $V$, and an $\mathbb{R}$-algebra homomorphism $\Phi$ of $\operatorname{dom}\left(v_{\delta}\right) \subset \mathcal{F}$ into $\operatorname{End}_{\mathbb{R}}(V)$ associated with the operator $A$, giving vectors $v_{01}, v_{10}, h_{a}, \Phi^{n} v_{\delta}=\Phi(Y)^{n} v_{\delta}(Y)(Y>0)$ in $V$ so that axioms (INT1) to (INT3) of the abstract intersection theory hold.

Proof. (ii) $\Longrightarrow$ (i): Suppose that the $\mathrm{RH}$ for $A$ does not hold. Then by (OP5-a) one can find and fix $Y>0$ so that $\sigma_{Y}(A)$ as described in Section 2 contains $s_{\alpha}, s_{\beta} \in \sigma(A)$ with $\operatorname{Re}\left(s_{\alpha}\right)<\frac{1}{2}, \operatorname{Re}\left(s_{\beta}\right)>\frac{1}{2}$. Therefore $\sigma_{Y}(A)$ contains $s_{1}$ such that $q^{\operatorname{Re}\left(s_{1}\right)}>q^{\frac{1}{2}}$, where $q=q(Y)$. Actually, if $0<q<1$ (re)set $s_{1}=s_{\alpha}$, while if $q>1$ (re)set $s_{1}=s_{\beta}$. 
Recall that $\sigma_{Y}(A)$ is a finite set. Let $s_{j}\left(2 \leq j \leq 2 g=2 g(Y)=\operatorname{dim} H^{\phi_{Y}}\right)$ be all the other eigenvalues of $A$ in $\sigma_{Y}(A)$, counted with algebraic multiplicities. (Note that Image $\left(P_{\sigma_{Y}(A)}\right)=H^{\phi_{Y}}$ since $\phi_{Y}(s) \neq 0$ for $s \in \sigma_{Y}(A)$.) Let $\lambda_{j}=\phi_{Y}\left(s_{j}\right)=q^{s_{j}}(1 \leq j \leq 2 g)$. Then by Lemma 3.4, $\nu_{n}=\sum_{j=1}^{2 g} \lambda_{j}^{n}$ is not $O\left(q^{\frac{n}{2}}\right)$, since we could choose $s_{1}$ so that $\left|\lambda_{1}\right|^{n}=\left|q^{s_{1}}\right|^{n}=q^{\frac{n}{2}}(1+\epsilon)^{n}$ for some $\epsilon>0$. By the spectral mapping theorem, $\sigma\left(\phi_{Y}(A)^{n}\right)=\sigma_{p}\left(\phi_{Y}(A)^{n}\right)=$ $\phi_{Y}\left(\sigma_{Y}(A)\right)^{n} \cup\{0\}=\left\{\lambda_{j}^{n} ; 1 \leq j \leq 2 g\right\} \cup\{0\}$. However, by (INT3), the Cauchy-Schwarz inequality and (IP-g), we see that $\nu_{n}$ is $O\left(q^{\frac{n}{2}}\right)$. This is a contradiction.

(i) $\Longrightarrow$ (ii): By Lemma 3.2, we have (IP-a) to (IP-f) and (INT3) for vectors in $V_{1}$ and $\Phi=\Phi(Y)$. All we have to do now is to verify (IP-g). Let us take a constant $q>1$ so that $q=q(Y)$ for all $Y>0$. If the $\mathrm{RH}$ for the operator $A$ holds, then each eigenvalue $\lambda_{\ell}(1 \leq \ell \leq 2 g)$ besides 0 , counted with algebraic multiplicities, of $\phi_{Y}(A)$ can be written as $\lambda_{\ell}=q^{\frac{1}{2}} e^{i \theta_{\ell}}\left(\theta_{\ell} \in\right.$ $\mathbb{R})$. By (OP3-b) one can choose eigenvectors $w_{\ell}$ associated with $\lambda_{\ell}$ so that $\phi_{Y}(A) w_{\ell}=\lambda_{\ell} w_{\ell}$. Let $\left\{e_{j}\right\}_{j=1}^{2 g}$ be an orthonormal basis of $H^{\phi_{Y}}$. Now one can write $e_{j}$ as $e_{j}=\sum_{\ell=1}^{2 g} \alpha_{j \ell} w_{\ell}$ for some $\alpha_{j \ell}=\alpha_{j \ell}(Y) \in \mathbb{C}$. Then in a similar way as in the proof of Lemma 3.2 (ii),

$$
\begin{gathered}
\left\langle\Phi(Y)^{n} v_{\delta}(Y), \Phi(Y)^{n} v_{\delta}(Y)\right\rangle_{V}=\sum_{j=1}^{2 g}\left\langle\phi_{Y}(A)^{n} e_{j}, \phi_{Y}(A)^{n} e_{j}\right\rangle_{H^{\phi_{Y}}} \\
=\sum_{j=1}^{2 g}\left\langle\sum_{\ell=1}^{2 g} \alpha_{j \ell} \phi_{Y}(A)^{n} w_{\ell}, \sum_{m=1}^{2 g} \alpha_{j m} \phi_{Y}(A)^{n} w_{m}\right\rangle_{H^{\phi_{Y}}} .
\end{gathered}
$$

Since $\phi_{Y}(A)^{n} w_{\ell}=\lambda_{\ell}^{n} w_{\ell}$, we have (IP-g). Therefore by Lemma 3.3, we have (INT1) and (INT2) for a subspace $V \subset V_{1}$. We have of course (INT3) by restricting to $V$.

Remark 3.1. Note that (OP3-b) is used only in the proof of (i) $\Longrightarrow$ (ii). To prove the $\mathrm{RH}$ for $A$ from the existence of an abstract intersection theory one does not need (OP3-b).

As mentioned in Section 2, the operator $A_{0}$ constructed in [11] satisfies all the conditions except for (OP3-b). Thus we have the following corollary.

Corollary 3.1. Assume that the operator $A_{0}$ in [11, Theorem 4.1] satisfies additionally the condition (OP3-b), or equivalently, the order of every nontrivial zero of the corresponding Dirichlet L-function is one. Then the following conditions are equivalent. 
(i) The Riemann Hypothesis holds for $A_{0}$ (and for the corresponding Dirichlet L-function).

(ii) There exists an abstract intersection theory as described in Theorem 3.1 for $A_{0}$.

\section{Analogy with the intersection theory on a surface over $\mathbb{F}_{q}$}

Let $C$ be a smooth projective curve over a finite field $\mathbb{F}_{q}$, and $S=C \times C$ the surface over $\mathbb{F}_{q}$. Let $\operatorname{Pic}(S)\left(\simeq H^{1}\left(S, \mathcal{O}_{S}^{\times}\right)\right)$be its Picard group, which we regard as a $\mathbb{Z}$-module, so as to preserve the analogy with Weil divisors. $V$ in Section 3 is modeled on $\operatorname{Pic}(S) \otimes_{\mathbb{Z}} \mathbb{R}$, and $\beta(\cdot, \cdot)$ in Section 3 is modeled on the $\mathbb{R}$-tensored intersection pairing $i(\cdot, \cdot)$ on $\operatorname{Pic}(S) \otimes_{\mathbb{Z}} \mathbb{R} . \Phi=\Phi(Y)$ in (INT1) is an analog of the linear mapping on $\operatorname{Pic}(S) \otimes_{\mathbb{Z}} \mathbb{R}$ induced by the morphism Frob $\times$ id on $S$. Then one may regard $v_{01}, v_{10}, v_{\delta}$ and $\Phi^{n} v_{\delta}$ in (INT1) as analogs of cycles pt $\times C, C \times \mathrm{pt}, \Delta$ and $\Gamma_{\mathrm{Frob}^{n}}$, respectively. The Hodge property comes from the Hodge index theorem. A Hodge vector $h_{a}$ corresponds to an (ample) hyperplane section of $S$, thereby $\beta\left(\cdot, h_{a}\right)$ gives an analog of the degree function $\operatorname{deg} \otimes_{\mathbb{Z}} 1: \operatorname{Pic}(S) \otimes_{\mathbb{Z}} \mathbb{R} \rightarrow \mathbb{R}$. Lemma 3.1 is an analog of Castelnuovo's inequality.

If $\varphi=$ Frob $^{n}$, then it turns out that

$$
\operatorname{tr}\left(\left.\varphi^{*}\right|_{H_{\mathrm{et}}^{0}}\right)=1=i\left(\Gamma_{\varphi}, \mathrm{pt} \times C\right) i(\Delta, C \times \mathrm{pt})
$$

and

$$
\operatorname{tr}\left(\left.\varphi^{*}\right|_{H_{\text {ét }}^{2}}\right)=q^{n}=i\left(\Gamma_{\varphi}, C \times \mathrm{pt}\right) i(\Delta, \mathrm{pt} \times C)
$$

So the Lefschetz fixed-point formula for the $\ell$-adic cohomology reads for $\varphi=$ Frob $^{n}$ as

$$
\begin{aligned}
\operatorname{tr}\left(\left.\varphi^{*}\right|_{H_{\text {ét }}^{1}}\right) & =i\left(\Gamma_{\varphi}, \mathrm{pt} \times C\right) i(\Delta, C \times \mathrm{pt})+i\left(\Gamma_{\varphi}, C \times \mathrm{pt}\right) i(\Delta, \mathrm{pt} \times C)-i\left(\Gamma_{\varphi}, \Delta\right) \\
& =:\left\langle\Gamma_{\varphi}, \Delta\right\rangle_{\operatorname{Pic}(S) \otimes_{\mathbb{Z}} \mathbb{R}}
\end{aligned}
$$

(INT3) is modeled on this, and $\phi(A)^{n}$ acting on $H$ is an analogy of $\left.\varphi^{*}\right|_{H_{\text {ét }}^{1}}$ acting on the first $\ell$-adic étale cohomology group $H_{\text {ét }}^{1}\left(C \otimes_{\mathbb{F}_{q}} \overline{\mathbb{F}}_{q}, \mathbb{Q}_{\ell}\right)$.

Introducing a cutoff function $\phi$ is modeled on Weil's explicit formula [1952b], [1972] in [12]. 


\section{Acknowledgment}

The first author was supported by the research grant of the Polish Ministry of Science and Education N N201 607440.

\section{References}

[1] A. Connes and M. Marcolli, Noncommutative geometry, quantum fields and motives, AMS Colloquium Publications, 55, AMS, Providence, RI; Hindustan Book Agency, New Delhi, 2008

[2] C. Deninger, Some analogies between number theory and dynamical systems on foliated spaces, Proceedings of the ICM (Berlin, 1998), I, Doc. Math. J. DMV, 1998, 163-186.

[3] I. Gohberg, S. Goldberg and M. A. Kaashoek, Classes of linear operators, I, Oper. Theory Adv. Appl. 49, Birkhäuser, Basel, 1990.

[4] A. Grothendieck, Sur une note de Mattuck-Tate, J. Reine Angew. Math. 200 (1958), 208-215.

[5] P. D. Lax and R. S. Phillips, Scattering Theory for Automorphic Functions, Ann. Math. Studies, 87, Princeton University Press, Princeton, 1976.

[6] P. Monsky, P-Adic analysis and Zeta functions, Lect. Math. 4, Kyoto University, Kinokuniya Book-Store, Tokyo, 1970.

[7] B. S. Pavlov and L. D. Faddeev, Scattering theory and automorphic functions, Zap. Naučn. Sem. Leningrad. Otdel. Mat. Inst. Steklov. (LOMI) 27 (1972), 161-193 (Russian); Engl. Transl.: J. Soviet Math. 3 (1975), 522-548.

[8] W. Rudin, Real and complex analysis, Second Ed., McGraw-Hill, New York, 1974.

[9] I. E. Segal, Irreducible representations of operator algebras, Bull. Amer. Math. Soc. 53 (1947), 73-88.

[10] J.-P. Serre, Analogues Kählériens de certaines conjectures de Weil, Ann. Math. 71 (1960), 392-394.

[11] Y. Uetake, Spectral scattering theory for automorphic forms, Integral Equations Operator Theory 63 (2009), 439-457. 
[12] A. Weil, Euvres Scientifiques Collected Papers, Intersection theory: (1940b), (1941), (1942), (1946a), (1948a,b) (vol. I): Explicit formulae: (1952b) (vol. II), (1972) (vol. III), Springer Verlag, New York, 1979 .

Faculty of Mathematics and Computer Science,

ADAM MiCKIEWICZ UNIVERSITY,

Ul. Umultowska 87, 61-614 Poznań, Poland

E-mail addresses: banaszak@amu.edu.pl; uetake@amu.edu.pl

ReCEIVED July 6, 2011 